Check for updates

Cite this: Chem. Commun., 2021, 57, 9838

Received 7th July 2021,

Accepted 3rd September 2021

DOI: $10.1039 / \mathrm{d} 1 \mathrm{cc} 03648 \mathrm{c}$

rsc.li/chemcomm

\section{Photo-crosslinking polymers by dynamic covalent disulfide bonds $\uparrow$}

\author{
Bianka Sieredzinska, ${ }^{a}$ Qi Zhang, (D ${ }^{a}$ Keimpe J. van den Berg, ${ }^{b}$ Jitte Flapper ${ }^{c}$ and \\ Ben L. Feringa (D)*a
}

\begin{abstract}
A simple and general strategy to construct photo-crosslinkable polymers by introducing sidechain 1,2-dithiolanes based on natural thioctic acid is presented. The disulfide five-membered rings act both as light-absorbing and dynamic covalent crosslinking units, enabling efficient photo-crosslinking and reversible chemical decrosslinking of polydimethylsiloxane polymers.
\end{abstract}

Crosslinking polymers by light provides many opportunities in order to develop smart manufacturing technologies for polymeric materials such as 3D laser printing, photo-patterning, microfabrication and coatings. ${ }^{1}$ Current major strategies include the introduction of photo-initiators which can form radicals to trigger the polymerizations and as a consequence, cure the linear polymers or resin by light irradiation. ${ }^{2}$ Although this common photocrosslinking strategy is efficient and well established, the inevitable residues of these photo-initiators usually accelerate the undesired aging process of the materials. ${ }^{3}$ The quest for the circularity and recycling of future materials demands reversibility of cross-linking (Fig. 1a), ${ }^{4}$ while material properties should not be compromised. Meanwhile, strategies like introducing photo-switchable molecules or structural units controlling supramolecular interactions have been developed to enable the light-controlled crosslinking/decrosslinking process of polymers. ${ }^{5}$ However, such photo-crosslinking strategies require chromophores that often need tedious synthesis while many of them are not robust because of the inherently weak nature of supramolecular interactions. ${ }^{6}$ Therefore, structurally simple units with inherent photo-crosslinking capability and featuring dynamic covalent bonds that support a robust crosslinked

\footnotetext{
${ }^{a}$ Stratingh Institute for Chemistry and Zernike Institute for Advanced Materials, Faculty of Science and Engineering, University of Groningen, Nijenborgh 4, 9747 AG Groningen, The Netherlands. E-mail: b.l.feringa@rug.nl

${ }^{b}$ Akzo Nobel Car Refinishes B.V., Rijksstraatweg 31, 2171 AJ Sassenheim, The Netherlands

${ }^{c}$ Akzo Nobel Decorative Coatings B.V., Rijksstraatweg 31, 2171 AJ Sassenheim, The Netherlands

$\dagger$ Electronic supplementary information (ESI) available. See DOI: 10.1039/ d 1 cc03648c
}

network as well as intrinsic reversibility is a fundamental challenge for chemists.

In order to develop a robust photo-crosslinking and decrosslinking strategy we focussed on 1,2-dithiolanes, a family of disulfide compounds with five-membered rings. ${ }^{7}$ Thioctic acid (TA) (Fig. 1b), a biologically occurring small-molecule 1,2-dithiolane, is particularly attractive ${ }^{8}$ featuring an intrinsic crosslinking and photoactive unit. In previous studies, our group has demonstrated the heat-induced ring-opening polymerization (ROP) of TA in a solventfree state to produce poly(TA) materials that can stretch, self-heal, stick to surfaces, and even be chemically recyclable in a closed-loop manner. ${ }^{9}$ Some early-stage studies explored the photochemistry of $\mathrm{TA}^{7 a, 10}$ concluding that the strained disulfide bonds can be scissored into diradicals by long-wavelength UV light, allowing to trigger the radical-initiated polymerization of TA. ${ }^{9,11}$ In other words, this photo-polymerization ability of TA is an inherent property, avoiding

a)
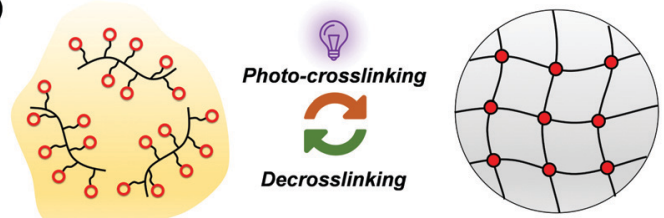

Flowing linear polymers

b)
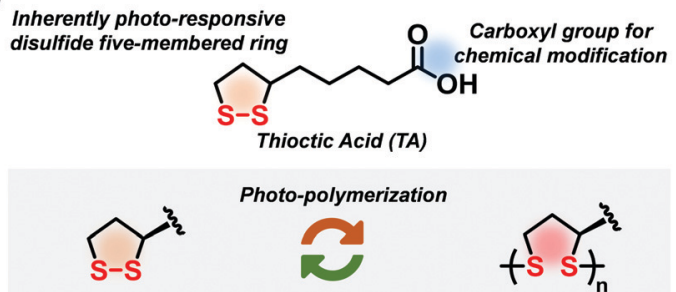

Photo-polymerization
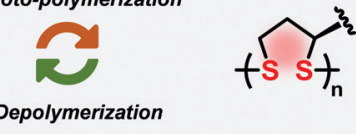

Fig. 1 (a) Conceptual illustration of the reversible photo-crosslinking strategy; (b) the structure of TA features inherently photo-responsive ability and enables reversible crosslinking mediated by dynamic covalent disulfide bonds. 
the need of introducing external photo-initiators or light-absorbers. To this end, we present here the use of this natural 1,2-dithiolanebased structure as a versatile dynamic covalent crosslinker for traditional polymers, to enable an inherently photo-crosslinkable, reversible and robust polymer network (Fig. 1).

Polydimethylsiloxane (PDMS) polymers were selected due to their optical transparency and these materials sustain a broad range of practical applications including coatings, elastomers and for photo-patterning manufacturing. ${ }^{12}$ To introduce TA as the side chain of PDMS, amine-modified PDMS (PDMS- $\mathbf{N H}_{2}$ ) (with $6-7 \%$ molar ratio of amine units) polymers were used to react with an NHS-activated TA ester (TA-NHS) (Fig. 2a). This simple post-modification reaction afforded over $80 \%$ yield, resulting in yellow and transparent polymer liquid PDMS-TA (For details on synthesis and characterization, see ESI $\dagger$ ). The resulting PDMS-TA polymer is highly soluble in many organic solvents and behaves like a viscous flowing liquid, indicating the uncrosslinked nature of linear polymers.

To characterize the polymer structure of the resulting PDMS-TA polymers, infrared (IR) spectroscopy was used (Fig. 2b). Compared with the initial PDMS-NH $\mathbf{N H}_{\mathbf{2}}$ polymers, the functionalized PDMS-TA polymers showed distinctive vibration bands of $\nu_{\mathrm{N}-\mathrm{H}}$ at $3300 \mathrm{~cm}^{-1}$ and $\nu_{\mathrm{C}=\mathrm{O}}$ at $1650 \mathrm{~cm}^{-1}$, indicating the presence of amide bonds. The ${ }^{1} \mathrm{H}$ NMR spectra of PDMS-TA further confirmed the distinctive proton signals of 1,2-dithiolanes in the polymers and the successful formation of amide bonds (Fig. 2c). Furthermore, considering the possibility that the amidation reactions might not proceed quantitatively, a typical amine probe molecule, 1-fluoro-2,4-dinitrobenzene (DNFB), was used to further quantify the functionalization ratio of PDMS-TA (Fig. S1, ESI $\dagger$ ). This analysis showed that the PDMS-TA polymers had no detectable free amine groups, indicating the complete post-modification reaction.

Next the photocrosslinking properties of the resulting PDMS-TA polymers were investigated (Fig. 3a). Using a UV lamp

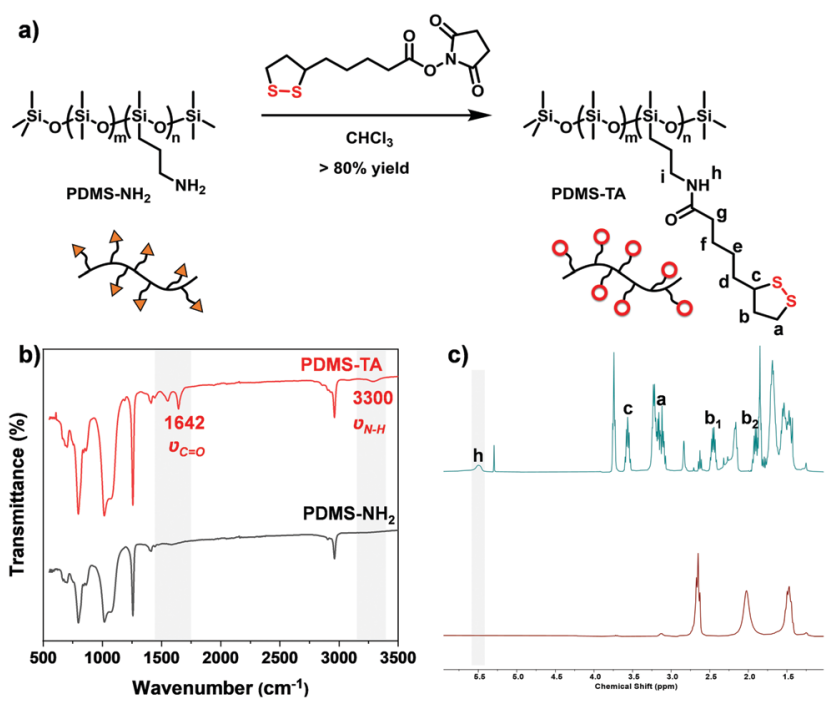

Fig. 2 (a) Post-modification preparation of PDMS-TA polymers; (b) IR spectra of the PDMS- $\mathrm{NH}_{2}$ and PDMS-TA polymers; (c) ${ }^{1} \mathrm{H}$ NMR spectra of the PDMS- $\mathrm{NH}_{2}$ and PDMS-TA polymers $\left(\mathrm{CDCl}_{3}, 400 \mathrm{MHz}, 298 \mathrm{~K}\right)$. a)

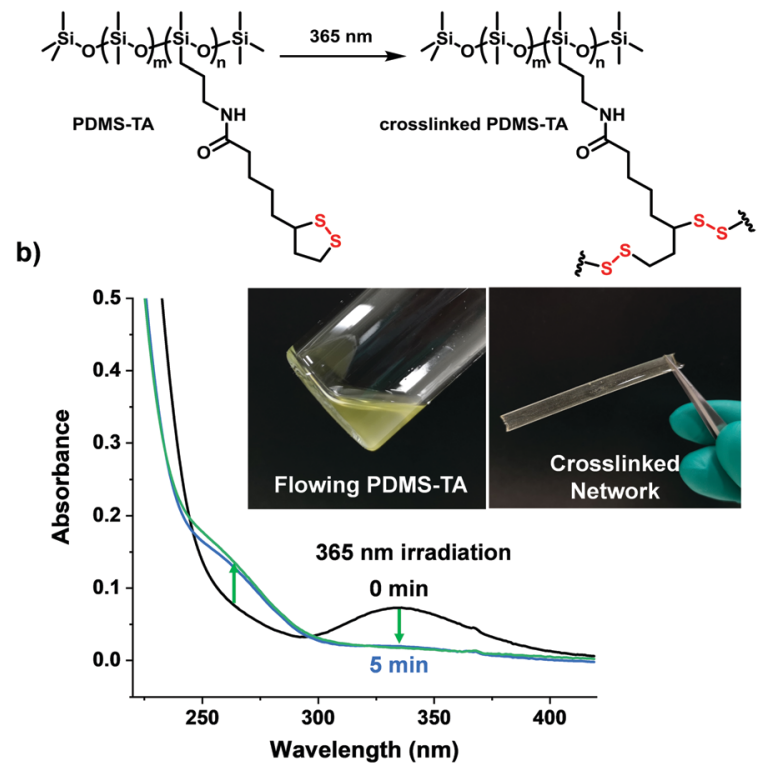

c)

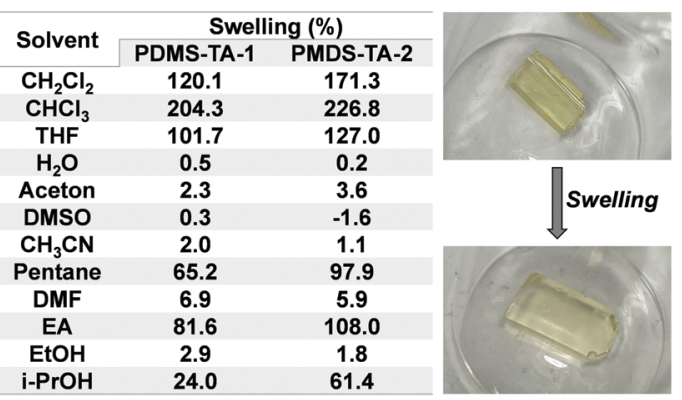

Fig. 3 (a) Photo-induced ring-opening crosslinking of the PDMS-TA polymers; (b) UV-Vis absorption spectra of the PDMS-TA polymer before and after photo-irradiation. Inset photographs of a typical PDMS-TA polymer before and after photo-crosslinking; (c) swelling properties of the formed crosslinked PDMS-TA network in different solvents at $298 \mathrm{~K}$. Inset images show a representative swollen sample in $\mathrm{CHCl}_{3}$.

with a wavelength of $365 \mathrm{~nm}$, the free flowing PDMS-TA liquid polymers were irradiated in a Teflon mould. After $1 \mathrm{~h}$ irradiation, transparent light yellow crosslinked materials were formed, which can be easily taken from the mould as freestanding and flexible solid samples (Fig. 3b). A similar method was also used to produce coatings on surfaces, based on this crosslinked PDMS network, with a typical contact angle of 105.8 degree (Fig. S2, ESI $\dagger$ ).

UV-Vis absorption spectra offered evidence of the successful ring-opening crosslinking driven by light irradiation: the distinctive absorption band of 1,2-dithiolanes at $330 \mathrm{~nm}$ was rapidly photobleached upon $20 \mathrm{~min}$ UV irradiation in the solvent-free state, and the simultaneously increased absorption of ring-opened species at 250-300 $\mathrm{nm}$ indicated the formation of polymers or oligomers in the network (Fig. 3b). Furthermore, upon irradiation in diluted solutions the presence of sulfur radicals was observed, based on their distinctive absorption band at $500 \mathrm{~nm}$ (Fig. S3, ESI $\dagger$ ), ${ }^{13}$ suggesting a photo-induced radical crosslinking mechanism. 
To establish the material properties of the crosslinked system, the swelling behaviour was investigated (Fig. 3c). The resulting PDMS-TA material exhibited typical swelling properties of a PDMS network with the high swelling ability in apolar solvents $\left(\mathrm{CHCl}_{3}, \mathrm{CH}_{2} \mathrm{Cl}_{2}\right.$, tetrahydrofuran, etc. $)$ and a low swelling ratio in polar solvents $\left(\mathrm{H}_{2} \mathrm{O}\right.$, dimethyl formamide, dimethylsulfoxide, etc.). The typical swelling behaviour and robust nature (no dissolution) indicated the successful covalent crosslinking of the material. Meanwhile, the materials also exhibited a thermal decomposition temperature above $200{ }^{\circ} \mathrm{C}$, indicating the robustness of the covalently crosslinked network (Fig. S4, ESI $\dagger$ ). The glass transition temperature of the materials is below $-20{ }^{\circ} \mathrm{C}$ according to differential scanning caborimetry (DSC) curves (Fig. S5, ESI $\dagger$ ), which is typical for PDMS polymers.

In order to compare the effect of polymer chain length, we synthesized two types of PDMS-TA polymers with different molecular weight, distinguished by the viscosity of $\mathbf{P D M S - \mathbf { N H } _ { 2 }}$ precursors i.e. PDMS-TA-1 and PDMS-TA-2 (additional details see ESI $\dagger$ ). The mechanical properties of the crosslinked PDMS-TA network were evaluated by tensile experiments (Fig. S6, ESI $†$ ). As a result, both materials exhibited typical tensile curves of a highly crosslinked polymeric network with notable Young's modulus, indicating the robust covalent crosslinking by the disulfide bonds. Due to the longer polymer chain, PDMS-TA-1 shows a higher Young's modulus $(9.79 \pm 0.46 \mathrm{MPa})$ than PDMS-TA-2 $(5.61 \pm 0.78 \mathrm{MPa})$, while the breaking elongation ratio of PDMS-TA-1 was slightly lower than that of PDMS-TA-2. The maximum strength of the two materials were similar due to the consistent crosslinking manner. Stress relaxation experiments showed steady storage modulus after relaxation (Fig. S7, ESI $\dagger$ ), indicating the robust and steady crosslinked network due to the covalent nature of disulfide bonds.

The photo-crosslinking ability of the PDMS-TA polymers also offered opportunities of designing photo-crosslinking adhesives (Fig. 4a). ${ }^{14}$ The uncrosslinked PDMS-TA polymers exhibited no adhesive ability for any surfaces because of the linear polymer chains showing free flowing behaviour. Irradiating the PDMS-TA polymer layer between two glass slides can cure the liquid layer in $1 \mathrm{~h}$, resulting in robust adhering two glass surfaces mediated by a thin and transparent crosslinked network (Fig. 4a and Fig. S8, ESI $\dagger$ ). Quantitative measurement gave the shear strength values of the crosslinked network being $0.20 \pm 0.09 \mathrm{MPa}$ for PDMS-TA-1, and $0.52 \pm 0.06 \mathrm{MPa}$ for PDMS-TA-2. The high shear strength suggest the excellent robustness of the crosslinked network especially considering the lack of (commonly required) polar groups such adhesive materials. Notably, the adhesive strength of PDMS-TA-2 was higher than that of PDMS-TA-1, which should be attributed to the higher penetration ability of shorter polymer chains that enables high contact area with the surface.

Finally we demonstrate that, due to the dynamic covalent nature of the disulfide bonds ${ }^{15}$ the resulting crosslinked network of PDMS-TA polymers can also be decrosslinked into linear polymers in the presence of chemical initiators, e.g. thiolate. In a typical decrosslinking experiment, $1 \%$ molar ratio of benzyl mercaptane and base 1,8-diazabicyclo[5.4.0]undec-7-ene (DBU)

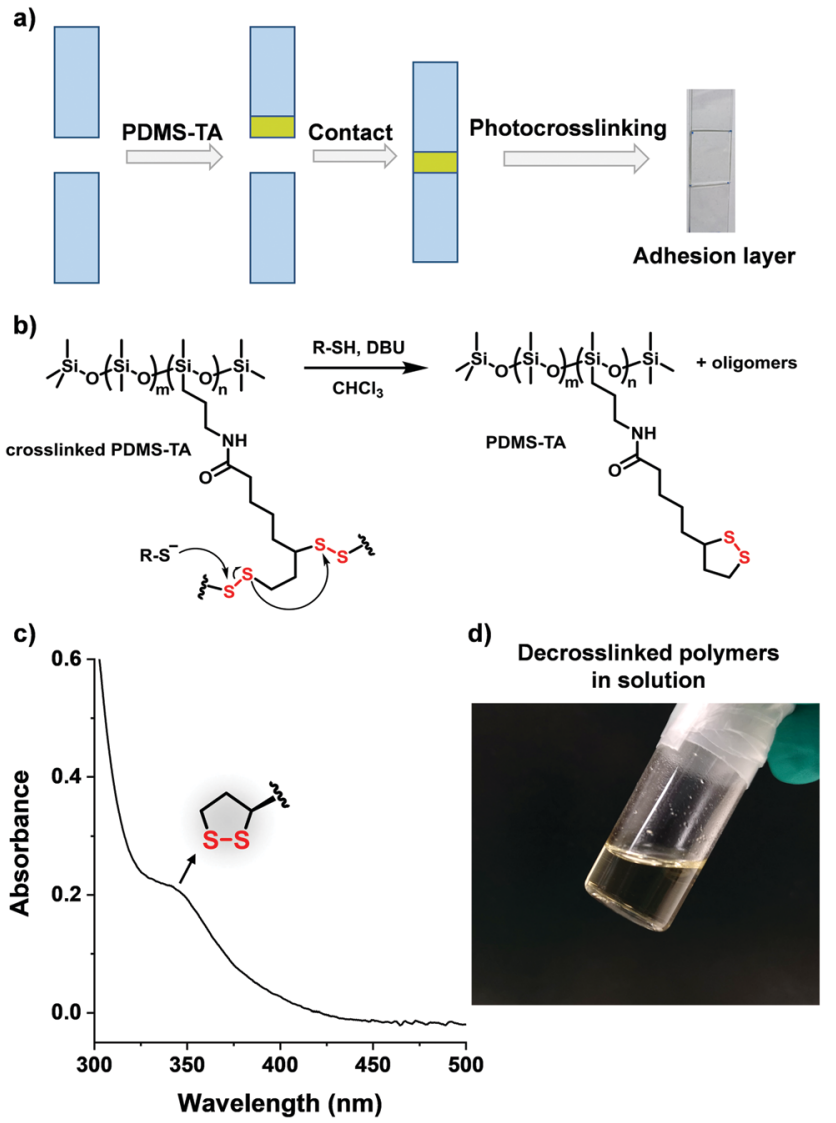

Fig. 4 (a) Schematic representation of the photo-crosslinking adhesive materials based on PDMS-TA polymers; (b) scheme of the chemically initiated decrosslinking of the network. The R-SH refers to benzyl mercaptane; (c) UV-Vis absorption spectra of the decrosslinked polymer solution in $\mathrm{CHCl}_{3}$. The emergence of the typical absorption of 1 , 2-dithiolanes indicated the successful decrosslinking; (d) photograph showing the completely dissolved network after decrosslinking.

were dissolved in $10 \mathrm{~mL} \mathrm{CHCl}_{3}$ solution to produce thiolate (Fig. 4b). Then a piece of PDMS-TA crosslinked network was immersed into the solution, and the mixture was stirred at room temperature. In sharp contrast with the insolubility of the crosslinked network in pure $\mathrm{CHCl}_{3}$, the solid polymer sample visibly dissolved in the presence of thiolate in $48 \mathrm{~h}$, forming a yellow transparent solution (Fig. $4 \mathrm{c}$ and d), which exhibited the distinctive absorption of the 1,2-dithiolanes monomer unit at $330 \mathrm{~nm}$, indicating the successful recovery of disulfide five-membered rings from the thiolate-initiated depolymerization of poly(disulfides). The ${ }^{1} \mathrm{H}$ NMR spectrum further supports the recovered distinctive absorptions of the five-membered rings $\left(\mathrm{H}_{\mathrm{a}}, \mathrm{H}_{\mathrm{b}}\right.$, and $\mathrm{H}_{\mathrm{c}}$ ) (Fig. S9, ESI $\dagger$ ). This preliminary experiment, showing mild catalytic depolymerisation, demonstrated the advantages of the dynamic covalent network based on TA towards chemically recyclable crosslinked polymers.

In conclusion, we demonstrated the successful photocrosslinking to form a polydimethylsiloxane network enabled by introducing 1,2-dithiolane units. The inherent photoresponsive ability of 1,2-dithiolanes enables a straightforward photo-crosslinking process in solvent-free state with the 
distinctive advantages that the external addition of photoinitiators or light-absorbers is not required. The resulting covalent crosslinked network can be also decrosslinked under ambient conditions in the presence of thiolate initiators due to the dynamic covalent nature of disulfide bonds. We expect that this proof-of-concept study based on biobased thioctic acid can provide a general and simple strategy for the design of reversible photo-crosslinking coatings and materials.

This work is part of the Advanced Research Center for Chemical Building Blocks, ARC CBBC, which is co-founded and co-financed by the Netherlands Organization for Scientific Research (NWO, contract 736.000.000) and the Netherlands Ministry of Economic Affairs and Climate. Q. Z. acknowledges the European Union's Horizon 2020 research and innovation programme under the Marie-Skłodowska-Curie grant agreement (101025041). The authors thank Prof. K. Loos and Ing. J. van Dijken at University of Groningen for the assistance with material characterization.

\section{Conflicts of interest}

There are no conflicts to declare.

\section{Notes and references}

1 (a) H. Xie, K. K. Yang and Y. Z. Wang, Prog. Polym. Sci., 2019, 95, 32-64; (b) K. S. Lim, J. H. Galarraga, X. Cui, G. C. Lindberg, J. A. Burdick and T. B. Woodfield, Chem. Rev., 2020, 120, 10662-10694; (c) H. Ge, W. Wu, Z. Li, G. Y. Jung, D. Olynick, Y. Chen, J. A. Liddle, S. Wang and R. S. Williams, Nano Lett., 2005, 5, 179-182; (d) A. Bagheri and J. Jin, ACS Appl. Polym. Mater., 2019, 1, 593-611; (e) N. Corrigan, J. Yeow, P. Judzewitsch, J. Xu and C. Boyer, Angew. Chem., Int. Ed., 2019, 58, 5170-5189; ( $f$ ) M. Layani, $\mathrm{X}$. Wang and S. Magdassi, Adv. Mater., 2018, 30, 1706344; (g) M. Regehly, Y. Garmshausen, M. Reuter, N. F. König, E. Israel, D. P. Kelly, C. Chou, K. Koch, B. Asfari and S. Hecht, Nature, 2020, 588, 620-624.

2 (a) C. R. Morgan, F. Magnotta and A. D. Ketley, J. Polym. Sci., Polym. Chem. Ed., 1977, 15, 627-645; (b) H. F. Gruber, Prog. Polym. Sci., 1992, 17, 953-1044; (c) K. T. Nguyen and J. L. West, Biomaterials, 2002, 23, 4307-4314; (d) J. Zhang and X. Pu, Polym. Chem., 2018, 9, 1530-1540; (e) G. Oster and N. L. Yang, Chem. Rev., 1968, 68, 125-151.

3 M. Panagiotopoulou, S. Beyazit, S. Nestora, K. Haupt and B. T. S. Bui, Polymer, 2015, 66, 43-51.

4 (a) J. B. Zimmerman, P. T. Anastas, H. C. Erythropel and W. Leitner, Science, 2020, 367, 397-400; (b) J. C. Worch and A. P. Dove, ACS Macro Lett., 2020, 9, 1494-1506; (c) M. Häußler, M. Eck,
D. Rothauer and S. Mecking, Nature, 2021, 590, 423-427; (d) L. T. Korley, T. H. Epps, B. A. Helms and A. J. Ryan, Science, 2021, 373, 66-69.

5 (a) D. H. Qu, Q. C. Wang, Q. W. Zhang, X. Ma and H. Tian, Chem. Rev., 2015, 115, 7543-7588; (b) X. Yan, F. Wang, B. Zheng and F. Huang, Chem. Soc. Rev., 2012, 41, 6042-6065; (c) F. Xu, L. Pfeifer, S. Crespi, F. K. C. Leung, M. C. Stuart, S. J. Wezenberg and B. L. Feringa, J. Am. Chem. Soc., 2021, 143, 5990-5997; (d) E. Weyandt, G. M. Ter Huurne, G. Vantomme, A. J. Markvoort, A. R. Palmans and E. W. Meijer, J. Am. Chem. Soc., 2020, 142, 6295-6303; (e) Y. L. Zhao and J. F. Stoddart, Langmuir, 2009, 25, 8442-8446; $(f)$ S. Matsumoto, S. Yamaguchi, S. Ueno, H. Komatsu, M. Ikeda, K. Ishizuka, Y. Iko, K. V. Tabata, H. Aoki, S. Ito, H. Noji and I. Hamachi, Chem. - Eur. J., 2008, 14, 3977-3986.

6 (a) D. B. Amabilino, D. K. Smith and J. W. Steed, Chem. Soc. Rev., 2017, 46, 2404-2420; (b) A. J. Savyasachi, O. Kotova, S. Shanmugaraju, S. J. Bradberry, G. M. O'Máille and T. Gunnlaugsson, Chemistry, 2017, 3, 764-811.

7 (a) J. A. Barltrop, P. M. Hayes and M. Calvin, J. Am. Chem. Soc., 1954, 76, 4348-4367; (b) L. J. Reed, B. G. DeBusk, I. C. Gunsalus and G. H. F. Schnakenberg, J. Am. Chem. Soc., 1951, 73, 5920.

8 U. Schmidt, P. Grafen and H. W. Goedde, Angew. Chem., Int. Ed. Engl., 1965, 4, 846-856.

9 (a) Q. Zhang, C. Y. Shi, D. H. Qu, Y. T. Long, B. L. Feringa and H. Tian, Sci. Adv., 2018, 4, eaat8192; (b) Q. Zhang, Y. X. Deng, H. X. Luo, C. Y. Shi, G. M. Geise, B. L. Feringa, H. Tian and D. H. Qu, J. Am. Chem. Soc., 2019, 141, 12804-12814; (c) Y. Deng, Q. Zhang, B. L. Feringa, H. Tian and D. H. Qu, Angew. Chem., Int. Ed., 2020, 59, 5278-5283; (d) Q. Zhang, Y. Deng, C. Y. Shi, B. L. Feringa, H. Tian and D. H. Qu, Matter, 2021, 4, 1352-1364.

10 (a) M. Calvin, J. Chem. Phys., 1955, 23, 1750; (b) M. Calvin, Proc. Natl. Acad. Sci. U. S. A., 1955, 41, 563.

11 (a) G. M. Scheutz, J. L. Rowell, S. T. Ellison, J. B. Garrison, T. E. Angelini and B. S. Sumerlin, Macromolecules, 2020, 53, 4038-4046; (b) C. Choi, J. L. Self, Y. Okayama, A. E. Levi, M. Gerst, J. C. Speros, C. J. Hawker, J. R. de Alaniz and C. M. Bates, J. Am. Chem. Soc., 2021, 143, 9866-9871.

12 (a) B. H. Jo, L. M. Van Lerberghe, K. M. Motsegood and D. J. Beebe, J. Microelectromech. Syst., 2000, 9, 76-81; (b) T. Fujii, Microelectron. Eng., 2002, 61, 907-914.

13 F. Denes, M. Pichowicz, G. Povie and P. Renaud, Chem. Rev., 2014, 114, 2587-2693.

14 (a) Y.-M. Tseng, A. Narayanan, K. Mishra, X. Liu and A. Joy, ACS Appl. Mater. Interfaces, 2021, 13, 29048-29057; (b) L. Kortekaas, J. Simke, D. W. Kurka and B. J. Ravoo, ACS Appl. Mater. Interfaces, 2020, 12, 32054-32060; (c) C. Y. Shi, Q. Zhang, H. Tian and D. H. Qu, SmartMat, 2020, 1, e1012.

15 (a) X. Zhang and R. M. Waymouth, J. Am. Chem. Soc., 2017, 139, 3822-3833; (b) G. A. Barcan, X. Zhang and R. M. Waymouth, J. Am. Chem. Soc., 2015, 137, 5650-5653; (c) Y. Liu, Y. Jia, Q. Wu and J. S. Moore, J. Am. Chem. Soc., 2019, 141, 17075-17080; (d) E. K. Bang, M. Lista, G. Sforazzini, N. Sakai and S. Matile, Chem. Sci., 2012, 3, 1752-1763; (e) E. K. Bang, G. Gasparini, G. Molinard, A. Roux, N. Sakai and S. Matile, J. Am. Chem. Soc., 2013, 135, 2088-2091. 